\title{
Rational Knots and a Theorem of Kanenobu
}

\author{
Alexander Stoimenow
}

\section{Introduction}

2. A Systematic Collection of Examples

3. An Empirical Approach

4. Some More Series of Examples

5. Questions

References
We give examples where Kanenobu's necessary condition for the rationality of a knot is not sufficient, and show that such examples are atypical.

\section{INTRODUCTION}

This note is devoted to the following theorem:

Theorem 1.1 [Kanenobu 1989]. For a rational or 2bridge knot or link, the Jones polynomial $V$ and the Brandt-Lickorish-Millett-Ho polynomial $Q$ satisfy

$\left(-u-u^{-1}\right)\left(Q\left(-u-u^{-1}\right)-1\right)=2\left(V(u) V\left(u^{-1}\right)-1\right)$.

See [Jones 1985; Brandt et al. 1986; Ho 1985] for the definition of these polynomials.

Apart from its elegance, formula (*) attracted my attention in particular because it provides a simple criterion to decide about the nonrationality of a knot (apart from considering Schubert's classification [1956] or knot group arguments).

The converse of this criterion turns out not to be true; that is, $(*)$ is not a necessary and sufficient condition for rationality. Here we construct infinite series of knots that are nonrational, in fact even nonalternating, but for which $(*)$ is satisfied. These examples have been suggested by empirical calculations (explained subsequently), which nevertheless reveal $(*)$ to be a surprisingly powerful test.

\section{A SYSTEMATIC COLLECTION OF EXAMPLES}

The first series of examples we construct suggested by empirical calculations is basically due to Joan Birman. We denote by $\sigma_{i}$ the Artin braid group generators and by $\Delta=\sigma_{2} \sigma_{1} \sigma_{2}$ the square root of the generator of the center of the 3 strand braid group $B_{3}$, as well as by $[\alpha]$ the exponent sum of $\alpha$, and by $w, g$ and $c$ the writhe, genus and crossing number.
Supported by a DFG postdoc grant. AMS subject classification: 57M25 
Proposition 2.1. Let $\alpha \in B_{3}$ be a 3-braid of the form

(i) $\sigma_{1}^{6 k \mp 1} \sigma_{2}^{ \pm 1}$ or

(ii) $\sigma_{1}^{6 k+1} \sigma_{2}^{-1} \sigma_{1} \sigma_{2}^{-1}$,

for $k \in \mathbb{N}$ sufficiently large. Then the knot $K=$ $\left(\Delta^{4 k} \alpha^{-1}\right)^{r}$ satisfies $(*)$, but is nonrational, even nonalternating. Infinitely many knots arise this way.

The proof we give here is of knot-theoretical flavour, but a more generally applicable argument will be given later.

Proof. By [Birman 1985, proposition 2], $K$ and $\hat{\alpha}$ have the same HOMFLY polynomial $P$ [Freyd et al. 1985], so also in particular the same $V$ and $\Delta$ [Alexander 1928] polynomials. (Such pairs of knots will subsequently be called Birman pairs.) Since $[\alpha]=$ $\left[\Delta^{4 k} \alpha^{-1}\right]$ we have $Q(\hat{\alpha})=Q(K)$ by [Kanenobu 1989 , Theorem 2]. Since $\hat{\alpha}$ is evidently 2 -bridge, $(*)$ holds for $K$.

Assume that $K$ were alternating. Since $\hat{\alpha}$ is a closed alternating braid, $\Delta(K)=\Delta(\hat{\alpha})$ is a monic polynomial. But by [Murasugi 1963] (see also Corollary 5.3 of [Cromwell 1989]) such a link is fibered, and therefore, by Theorem A of [Murasugi 1991], the Morton-Williams-Franks inequality [Morton 1986; Franks and Williams 1987] is sharp on $K$.

If $\alpha$ is of type (i), $K$ would then be a $(2, n)$ torus link, and $V(K)=V(\hat{\alpha})$ would imply $K=\hat{\alpha}$. But (basically as observed by Birman) Murasugi's formulas $[1974, \S 9-11]$ show that $\sigma(K) \neq \sigma(\hat{\alpha})$ for $k$ sufficiently large, a contradiction.

If $\alpha$ is of type (ii), the (closed braid) diagram $\hat{\alpha}$ is reduced, and the Morton-Williams-Franks bound for both $K$ and $\hat{\alpha}$ is sharp and equals 3 . Then by [Murasugi 1991, Corollary 2] a reduced alternating diagram $D$ of $K$ must have the same crossing number as the diagram $\hat{\alpha}$. Therefore, since

$$
\begin{aligned}
g(D)=g(K) & =\max \operatorname{deg} \Delta(K) \\
& =\max \operatorname{deg} \Delta(\hat{\alpha})=g(\hat{\alpha}),
\end{aligned}
$$

the number of Seifert circles of $D$ is the same as this of $\hat{\alpha}$, namely 3 . But by Morton's inequalities [1986] the $P$ polynomial determines the writhe of a diagram of minimal number of Seifert circles, if the Morton-Williams-Franks inequality is sharp. Therefore $[\alpha]=w(\hat{\alpha})=w(D)$. Since $D$, as a diagram with 3 Seifert circles, can be made into a braid diagram by at most one Vogel move [Vogel
1990], and since $\sigma$ changes at most by 2 under a crossing change, the remark after Proposition 11.1 of [Murasugi 1974] shows that $|\sigma(K)-\sigma(\hat{\alpha})| \leq 2$. However, by Murasugi's signature formulas, for $k$ large enough $|\sigma(K)-\sigma(\hat{\alpha})|$ also gets large enough, a contradiction.

Finally, to show that infinitely many of the knots are distinct, let $k \rightarrow \infty$ and use again Murasugi's signature formulas showing $\sigma \rightarrow \infty$.

\section{AN EMPIRICAL APPROACH}

A more realistic estimate for the quality of $(*)$ as a rationality test can be obtained by examining the tables in [Hoste and Thistlethwaite 1999].

First, $(*)$ detected all nonrational prime knots from the tables of [Rolfsen 1976] (which are easy to identify from the Conway notation recorded there). For knots of 11 or more crossings, Thistlethwaite does not specify which knots in his tables are rational, but the number of such knots for given low crossing number can be obtained by computer in a few seconds by enumerating iterated fractions arising from compositions of the crossing number into the entries of the Conway notation, and considering (only) fractions $p / q$ with $p, q \in \mathbb{N}$ mutually prime and $p$ odd up to the equivalence $p / q_{1} \sim p / q_{2} \Longleftrightarrow$ $q_{1} q_{2}^{ \pm 1}= \pm 1$ in $\mathbb{Z}_{p}^{*}$ (see [Kanenobu 1986], for example). The numbers are

\begin{tabular}{|c|rrrrrr|}
\hline crossing number & 11 & 12 & 13 & 14 & 15 & 16 \\
\# of rational knots & 91 & 176 & 352 & 693 & 1387 & 2752 \\
\hline
\end{tabular}

A formula for these numbers has been proved in [Ernst and Sumners 1987].

Considering prime alternating knots, I found that the number of knots satisfying $(*)$ coincides up to 16 crossings with the one of the above table, showing that $(*)$ decides about rationality of any such knot.

A further check showed $(*)$ to be violated for any composite knot of at most 16 crossings, assuming that so far the crossing number is additive under connected sum and taking from a prime knot and its obverse only one as a factor, as mirroring a composite factor does not change either of the expressions on both hand-sides of $(*)$. By [Menasco 1984; Kidwell 1987; Thistlethwaite 1987], $(*)$ is easily shown to be violated by any alternating composite knot, by comparing the edge coefficients. 


\begin{tabular}{|l|rrrrrrrrrrrrrr|r|}
\hline \multicolumn{1}{|c|}{ knot } & \multicolumn{11}{c|}{ Description in the notation of [Dowker and Thistlethwaite 1983] } \\
\hline $12_{1879}$ & 4 & 12 & -18 & 14 & -20 & 2 & 8 & -22 & -24 & -10 & -6 & -16 & & \\
$12_{2037}$ & 6 & 10 & 14 & -18 & 2 & -20 & 4 & 22 & 24 & -8 & -12 & 16 & & \\
$13_{7750}$ & 4 & 12 & 14 & -16 & 18 & -20 & 2 & -22 & 24 & -26 & -6 & 8 & -10 & \\
$13_{7960}$ & 4 & 12 & 16 & -22 & 14 & -20 & 2 & 8 & 24 & 26 & -10 & -6 & 18 & \\
$14_{33787}$ & 4 & 12 & 16 & -14 & -22 & 2 & -20 & 24 & -26 & -28 & -10 & -8 & 6 & -18 \\
$14_{43535}$ & 6 & 10 & 24 & -18 & 2 & -20 & -22 & 26 & 28 & -8 & -12 & 4 & 14 & 16 \\
$14_{44370}$ & 6 & 12 & 16 & -22 & -18 & 4 & -24 & 2 & -26 & -8 & -28 & -14 & -10 & -20 \\
$14_{46672}$ & 8 & -10 & 12 & -18 & 20 & -22 & 24 & 26 & -6 & 28 & -2 & 4 & 16 & 14 \\
$14_{46862}$ & 8 & -12 & -16 & -20 & 22 & -2 & 24 & -4 & 26 & -6 & 28 & 10 & 14 & 18 \\
$15_{157719}$ & 4 & 12 & 14 & -16 & 18 & -28 & 2 & -22 & 24 & -26 & -30 & -6 & 8 & -10 & -20 \\
$15_{168643}$ & 4 & 12 & 18 & 14 & 24 & 22 & 2 & -26 & 6 & -28 & -30 & 10 & 8 & -16 & -20 \\
$15_{233158}$ & 6 & 12 & 16 & -24 & -20 & -26 & 4 & -28 & 2 & -10 & -8 & -30 & -18 & -14 & -22 \\
$15_{247180}$ & 6 & 16 & 14 & 20 & -26 & 18 & -24 & 4 & 2 & 10 & 28 & 30 & -12 & -8 & 22 \\
\hline
\end{tabular}

TABLE 1. Knots of up to 15 crossings for which Kanenobu's formula (*) fails as a rationality test.

It is clear that among the nonalternating knots examples should occur, and the simplest ones are two knots of 12 crossings, $12_{2037}$ and $12_{1879}$. The first of these forms a famous Birman pair (see [Lickorish and Millett 1987, Example 17]) with $7_{1}$; the coincidence of $Q$ in this case was observed, without further explanation, already in [Brandt et al. 1986]. The complete list of exceptions up to 15 crossings is given in Table 1.

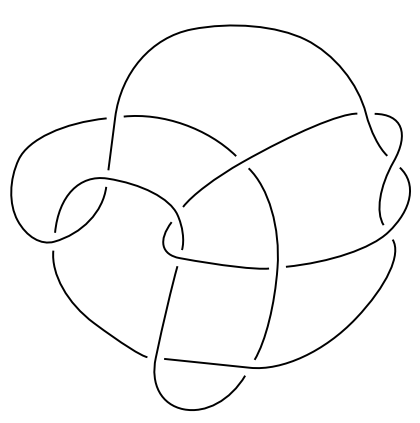

121879

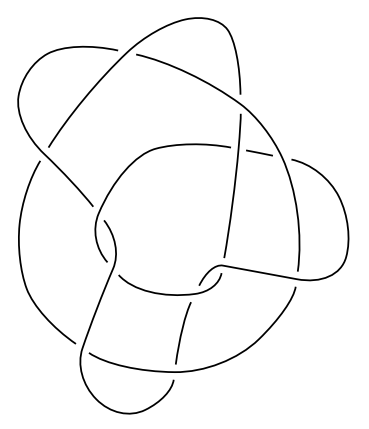

$12_{2037}$
FIGURE 1. The two simplest knots for which $(*)$ fails as rationality test.

Besides $12_{2037}$, Proposition 2.1 explains two more of these examples: $14_{43535}$ (associated to $10_{2}$ ) and $14_{46862}$ (associated to the $(2,11)$-torus knot $11_{367}$ ).

For all 13 knots listed in Table 1, nonalternation can be proved by the Kauffman [1990] $F$ polynomial. In fact, except for the Birman (pair) knots and the two further examples $13_{7960}$ and $15_{168643}$ (of which the first has the same $V$ and $Q$, but not $\Delta$, as $5_{2}$ ), already the criteria for $V$ given in [Kauffman 1987] and [Thistlethwaite 1987, Theorem 1] work.
The small number of exceptions compared to the total number of knots given in [Hoste et al. 1998] testifies to the quality of $(*)$ as a rationality test.

\section{SOME MORE SERIES OF EXAMPLES}

It may appear that the 10 knots of Table 1 that lie outside of the scope of Proposition 2.1 satisfy $(*)$ by accident. However, there are patterns underlying some of these remaining examples. Drawing the pictures, one observes striking similarities between some of them, which can be extended to infinite series.

Example 4.1. For example, the diagrams of $13_{7960}$ and $15_{247180}$ differ just by a $\bar{t}_{2}^{\prime}$ move at the crossing marked with an arrow in Figure 2. Applying further $\bar{t}_{2}^{\prime}$ moves we find that the next 8 diagrams still satisfy $(*)$. Thus we are lead to conjecture that this will hold for the whole series of diagrams.

This can be shown by some messy calculation, or by the following analytic argument. To simplify the notation, set $z=-u-u^{-1}$ and $z^{\prime}=\sqrt{z^{2}-4}$ from now on. We need to show that

$$
z(Q(z)-1)=2\left(V\left(\frac{z+z^{\prime}}{2}\right) V\left(\frac{z-z^{\prime}}{2}\right)-1\right)
$$

for the polynomials $Q_{i}$ and $V_{i}$ of the diagrams $D_{i}$ with $i$ twists. Considering the generating functions

$$
g(x, z):=\sum_{i=0}^{\infty} Q_{i}(z) x^{i}
$$


and

$$
f(x, y, z):=\sum_{i, j=0}^{\infty} V_{i}\left(\frac{z+z^{\prime}}{2}\right) \cdot V_{j}\left(\frac{z-z^{\prime}}{2}\right) x^{i} y^{j}
$$

(the series converge absolutely in a neighborhood of $(x, y, z)=(0,0,0)$ and $(x, z)=(0,0)$, respectively, because of the exponential growth of the polynomial coefficients in the crossing number) we find by the relations of the $Q$ and $V$ polynomials that these are rational functions in $x$ and $y$ (with coefficients in the fraction field $\mathcal{F}$ of $\mathbb{Z}\left[z, z^{\prime}\right]$ ) whose denominators have the form

$$
g(x, z)=\frac{g_{1}(x, z)}{g_{2}(x, z)}
$$

with $\operatorname{deg}_{x} g_{2} \leq 3$ and $(1-x) \mid g_{2}$ in the case of $g$ and $f(x, y, z)=\frac{P(x, y, z)}{(1-x)(1-y)\left(\left(z+z^{\prime}\right)^{2} x-4\right)\left(\left(z-z^{\prime}\right)^{2} y-4\right)}$

with $P \in \mathcal{F}[x, y], \operatorname{deg}_{x} P \leq 2, \operatorname{deg}_{y} P \leq 2$ in the case of $f$. To extract the diagonal part $i=j$ of $f$ that we are interested in (we call this 'contracting'), we apply a usual trick from harmonic analysis, obtaining

$$
\begin{aligned}
\tilde{f}(t, z) & =\int_{0}^{1} f\left(\sqrt{t} e^{2 \pi i u}, \sqrt{t} e^{-2 \pi i u}, z\right) d u \\
& =\frac{1}{2 \pi i} \oint_{|u|=\sqrt{t}} \frac{1}{u} f\left(u, \frac{t}{u}, z\right) d u .
\end{aligned}
$$

The path integral can be calculated by evaluating the relevant residues for small $t$, namely

$$
u=0, \quad u=t, \quad u=t\left(\frac{z-z^{\prime}}{2}\right)^{2},
$$

obtaining rational expressions in $t$ with denominators composed by the five factors $t^{2}, t-1$,

$$
\left(\frac{z+z^{\prime}}{2}\right)^{2} t-1, \quad\left(\frac{z-z^{\prime}}{2}\right)^{2} t-1, \quad\left(\frac{z+z^{\prime}}{z-z^{\prime}}\right)^{2} t-1,
$$

the numerators being of at most the same degree in $t$ as the denominators.

Thus the identity we wish to show, namely

$$
\frac{1}{1-t}+\frac{z}{2}\left(g(t, z)-\frac{1}{1-t}\right)=\tilde{f}(t, z)
$$

when multiplied by the lowest common multiple of the denominators (which is of degree 8 in $t$ ), turns into a polynomial identity of degree at most 8 in $t$.
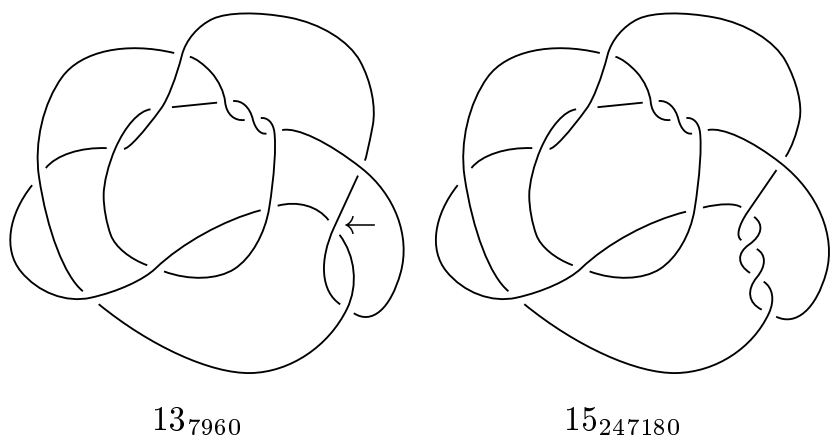

FIGURE 2. Two examples of nonalternating knots satisfying $(*)$, differing by a $\bar{t}_{2}^{\prime}$ move only.

To prove the identity, then, it suffices to show equality for the first 9 coefficients in the Taylor expansion, which correspond to the first 9 diagrams, the ones we checked above.

Example 4.2. The knots $13_{7750}$ and $15_{157719}$ in Figure 3 differ just by two local replacements of a crossing by a parallel clasp. If we repeat this procedure, adding the same number of crossings at both places, we obtain 6 further diagrams satisfying $(*)$.

Again, considering this series as the diagonal part of the 2-parameter series $D_{i, i^{\prime}}$ (with respectively $i$ and $i^{\prime}$ half-twists inserted: note that for $i+i^{\prime}$ odd these are 2 component link diagrams), one can build

$$
\begin{aligned}
& f\left(x, x_{1}, y, y_{1}, z\right):= \\
& \sum_{i, j, i^{\prime}, j^{\prime} \geq 0} V_{i, i^{\prime}}\left(\frac{z \pm z^{\prime}}{2}\right) V_{j, j^{\prime}}\left(\frac{z \pm z^{\prime}}{2}\right) x^{i} x_{1}^{i^{\prime}} y^{j} y_{1}^{j^{\prime}}
\end{aligned}
$$

and contract three times, obtaining a polynomial in $t$ with coefficients in some higher-degree algebraic
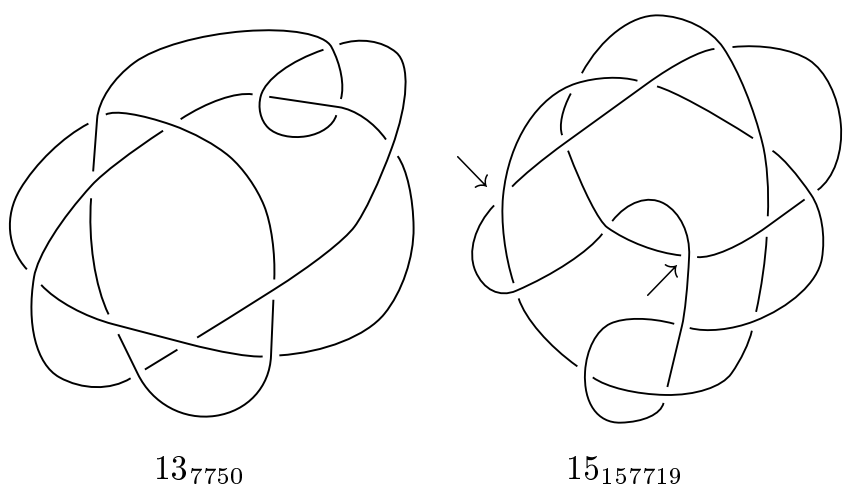

$15_{157719}$

FIGURE 3. Two further similar examples: smoothing out the marked crossings on the right gives the knot with the diagram on the left. 
extension of $\mathcal{F}$; likewise one builds the corresponding series for $Q$,

$$
g\left(x, x_{1}, z\right):=\sum_{i, i^{\prime} \geq 0} Q_{i, i^{\prime}}(z) x^{i} x_{1}^{i^{\prime}}
$$

and contracts once. One can then show the general case by some finite number of checks (or by some even messier direct calculation).

Instead we show that the knots $D_{i, j}$ are nonalternating (something we would need to show also in the previous example, but which is then a special case of the argument given in the following lines).

We consider the maximal $z$-degree of the Kauffman polynomial and check that for $p+q=6,7$, with $p, q>0$, it is $c\left(D_{p, q}\right)-4$, and that the maximal coefficient of $z$ is of the form $\pm a^{k} \mp a^{k+4}$ for some $k \in \mathbb{Z}$, which exhibits nonalternation [Kauffman 1990, p. 426-427]. For $p+q>7$, with $p, q>0$, the same property follows by induction on $p+q$ by applying the Kauffman relation near a crossing $p$ in the box with $q$ twists with $q \geq p$ and using the general inequality

$$
\max \operatorname{deg}_{z} F(K) \leq c(K)-1
$$

for any nontrivial link $K$, applying it on the diagram on which the crossings in the twist box have become nugatory.

There is a further similarity of diagrams between $14_{44370}$ and $15_{233158}$, this time involving local changes at 3 crossings; but in this case I was not able to extend it to an infinite series.

\section{QUESTIONS}

We conclude with a summary of the problems suggested by empirical evidence.

Question 5.1. Is there a composite knot satisfying $(*)$ ?

Question 5.2. Is there a nonrational alternating knot which satisfies $(*)$ (it would need to be prime)?

Question 5.3. Is there a nonrational knot with the $F$ polynomial of a rational knot?

Among rational knots (and also nonrational ones [Lickorish 1988]), duplications of $F$ are well-known and have been tabulated by Kanenobu.

\section{REFERENCES}

[Alexander 1928] J. W. Alexander, "Topological invariants of knots and links", Trans. Amer. Math. Soc. 30:2 (1928), 275-306.

[Birman 1985] J. S. Birman, "On the Jones polynomial of closed 3-braids", Invent. Math. 81:2 (1985), 287-294.

[Brandt et al. 1986] R. D. Brandt, W. B. R. Lickorish, and K. C. Millett, "A polynomial invariant for unoriented knots and links", Invent. Math. 84:3 (1986), 563-573.

[Cromwell 1989] P. R. Cromwell, "Homogeneous links", J. London Math. Soc. (2) 39:3 (1989), 535-552.

[Dowker and Thistlethwaite 1983] C. H. Dowker and M. B. Thistlethwaite, "Classification of knot projections", Topology Appl. 16:1 (1983), 19-31.

[Ernst and Sumners 1987] C. Ernst and D. W. Sumners, "The growth of the number of prime knots", Math. Proc. Cambridge Philos. Soc. 102:2 (1987), 303-315.

[Franks and Williams 1987] J. Franks and R. F. Williams, "Braids and the Jones polynomial", Trans. Amer. Math. Soc. 303:1 (1987), 97-108.

[Freyd et al. 1985] P. Freyd, D. Yetter, J. Hoste, W. B. R. Lickorish, K. Millett, and A. Ocneanu, "A new polynomial invariant of knots and links", Bull. Amer. Math. Soc. (N.S.) 12:2 (1985), 239-246.

[Ho 1985] C. F. Ho, "A polynomial invariant for knots and links: preliminary report", Abstracts Amer. Math. Soc. 6 (1985), 300.

[Hoste and Thistlethwaite 1999] J. Hoste and M. Thistlethwaite, "KnotScape", knot polynomial calculation software, 1999. See http://www.math.utk.edu/ $\sim$ morwen/knotscape.html.

[Hoste et al. 1998] J. Hoste, M. Thistlethwaite, and J. Weeks, "The first 1,701,936 knots", Math. Intell. 20:4 (1998), 33-48.

[Jones 1985] V. F. R. Jones, "A polynomial invariant for knots via von Neumann algebras", Bull. Amer. Math. Soc. (N.S.) 12:1 (1985), 103-111.

[Kanenobu 1986] T. Kanenobu, "Examples on polynomial invariants of knots and links", Math. Ann. 275:4 (1986), 555-572.

[Kanenobu 1989] T. Kanenobu, "Relations between the Jones and $Q$ polynomials for 2-bridge and 3-braid links", Math. Ann. 285:1 (1989), 115-124.

[Kauffman 1987] L. H. Kauffman, "State models and the Jones polynomial", Topology 26:3 (1987), 395-407. 
[Kauffman 1990] L. H. Kauffman, "An invariant of regular isotopy", Trans. Amer. Math. Soc. 318:2 (1990), 417-471.

[Kidwell 1987] M. E. Kidwell, "On the degree of the Brandt-Lickorish-Millett-Ho polynomial of a link", Proc. Amer. Math. Soc. 100:4 (1987), 755-762.

[Lickorish 1988] W. B. R. Lickorish, "The panorama of polynomials for knots, links and skeins", pp. 399-414 in Braids (Santa Cruz, CA, 1986), edited by J. S. Birman and A. L. Libgober, Contemp. Math. 78, Amer. Math. Soc., Providence, RI, 1988.

[Lickorish and Millett 1987] W. B. R. Lickorish and K. C. Millett, "A polynomial invariant of oriented links", Topology 26:1 (1987), 107-141.

[Menasco 1984] W. Menasco, "Closed incompressible surfaces in alternating knot and link complements", Topology 23:1 (1984), 37-44.

[Morton 1986] H. R. Morton, "Seifert circles and knot polynomials", Math. Proc. Cambridge Philos. Soc. 99:1 (1986), 107-109.
[Murasugi 1963] K. Murasugi, "On a certain subgroup of the group of an alternating link", Amer. J. Math. 85 (1963), 544-550.

[Murasugi 1974] K. Murasugi, On closed 3-braids, Memoirs Amer. Math. Soc. 151, Amer. Math. Soc., Providence, 1974.

[Murasugi 1991] K. Murasugi, "On the braid index of alternating links", Trans. Amer. Math. Soc. 326:1 (1991), 237-260.

[Rolfsen 1976] D. Rolfsen, Knots and links, Mathematics Lecture Series 7, Publish or Perish, Berkeley, 1976. Reprinted with corrections, Publish or Perish, Houston, 1990.

[Schubert 1956] H. Schubert, "Knoten mit zwei Brücken", Math. Z. 65 (1956), 133-170.

[Thistlethwaite 1987] M. B. Thistlethwaite, "A spanning tree expansion of the Jones polynomial", Topology 26:3 (1987), 297-309.

[Vogel 1990] P. Vogel, "Representation of links by braids: a new algorithm", Comment. Math. Helv. 65:1 (1990), 104-113.

Alexander Stoimenow, Max-Planck-Institut für Mathematik, Vivatsgasse 7, D-53111 Bonn, Germany (alex@mpim-bonn.mpg.de, http://guests.mpim-bonn.mpg.de/alex)

Received January 14, 2000; accepted in revised form July 8, 2000 